\title{
Design Second Order Vibration Reduction
}

\author{
Meysam Kazeminasab, Zahra Esmaeili, Alireza Salehi, Mahdi Mirshekaran and \\ Farzin Piltan \\ Intelligent System and Robotic Lab, Iranian Institute of Advance Science and \\ Technology (IRAN SSP), Shiraz/Iran \\ Email: piltan_f@iranssp.com,WWW.IRANSSP.COM/english
}

\begin{abstract}
Design of a robust controller for multi input-multi output (MIMO) nonlinear uncertain dynamical system can be a challenging work. This paper focuses on the design and analysis of a high performance adaptive baseline sliding mode control for second order nonlinear uncertain system, in presence of uncertainties to reduce the vibration. In this research, sliding mode controller is a robust and stable nonlinear controller which selected to control of robot manipulator. The proposed approach effectively combines of design methods from switching sliding mode controller, adaptive model-free baseline controller and linear Proportional-Derivative (PD) control to improve the performance, stability and robustness of the sliding mode controller. Sliding mode controller has two important subparts, switching and equivalent. Switching part (discontinuous part) is very important in uncertain condition but it causes chattering phenomenon. To solve the chattering, the most common method used is linear boundary layer saturation method, but this method lost the stability. To reduce the chattering with respect to stability and robustness; linear controller is added to the switching part of the sliding mode controller. The linear controller is to reduce the role of sliding surface slope and switching (sign) function. The nonlinearity term of the sliding mode controller is used to eliminate the decoupling and nonlinear term of link's dynamic parameters. However nonlinearity term of sliding mode controller is very essential to reliability but in uncertain condition or highly nonlinear dynamic systems it can cause some problems. To solve this challenge the baseline controller is used as online tune or adaptive controller. This controller improves the stability and robustness, reduces the chattering as well and reduces the level of energy due to the torque performance as well.
\end{abstract}

Keywords: Motor vibration, vibration control, variable structure control, baseline methodology, stability, robust, online tuning, chattering

\section{Introduction}

Design a robust controller for multi input-multi output (MIMO) nonlinear uncertain dynamical system (e.g., robot manipulator) is the main challenging work in this research. Robot manipulators are set of links which connected by joints, they are multi input and multi output (MIMO), nonlinear, time variant, uncertain dynamic systems and are developed either to replace human work in many fields such as in industrial or in the manufacturing. Complexities of the tasks caused to design mechanical architectures and robot manipulator with nonlinear behavior. These factors are:

- Time-varying parameters based on tear and ware.

- Simplifying suppositions in system modelling caused to have un-modelled dynamic parameters.

- External disturbance and noise measurement which it is caused to generate uncertainties. 
According to above discussion, robot manipulators are nonlinear uncertain systems with human-like behavior therefore, control of these systems are complicated. Robot manipulators are divided into two main groups, serial links robot manipulators and parallel links robot manipulators. OCTARM continuum robot manipulator is a serial link robot manipulator, in this type of robot links and joints are serially connected between base and end-effector [1-2]. Study of robot manipulators are classified into two main subjects: kinematics and dynamics. Study of kinematics is important to design controller in practical applications. Dynamic modeling of robot manipulator is used to illustrate the behavior of robot manipulator (e.g., MIMO, nonlinear, uncertain parameters and ...), design of nonlinear conventional controller such as conventional Computed Torque Controller and Sliding Mode Controller and also for simulation. It also used to explain some dynamic parameters effect to system behavior. According to the literature [1-8]. OCTARM continuum robot manipulator is a serial links, and highly nonlinear dynamic systems, which control of this system with linear behavior is the main challenge in this research.

To control of OCTARM continuum robot manipulator, three purposes are very important:

- Stability: Stability is due to the proper functioning of the system. A system is called stable if for any bounded input signal the system's output will stay bounded. Therefore limitation of output deviation is very important for any design.

- Robust: Robust method is caused to achieve robust and stable performance in the presence of uncertainty and external disturbance. A system is robust when it would also work well under different conditions and external disturbance.

- Reliability: to control of nonlinear and uncertain systems, reliability play important role and most of model-base controller are reliable [9-12].

As a result, design a controller based on these three factors are the main challenge in this work. Based on control theory; controllers for robot manipulators are divided into two main collections:

Conventional control theory and intelligent control theory where, conventional control theories are work based on nonlinear dynamic parameters of OCTARM continuum robot manipulator and these are divided into two main categories: Linear control method and nonlinear control method. Intelligent control theory is worked based on intelligent control theory and it is free of nonlinear dynamic parameters of robot manipulator. An Important challenge in this part is select the best methodology when lots of control techniques are accessible [13-16].

According to the dynamic formulation of OCTARM continuum robot manipulators, they are uncertain and there exist strong coupling effects between joints. The problem of coupling effects play important role to get best performance in robot manipulator. In linear controller this challenge can be reduced, with the following two methods:

- Limiting the performance of the system according to the required velocities and accelerations, but now the applications demand for faster and lighter robot manipulators.

- Using a high gear ratio (e.g., 250 to 1 ) at the mechanical design step, in this method the price paid is increased due to the gears.

Therefore linear type of controller, such as PD, PI or PID controllers cannot be having a good results and performance.

Conventional nonlinear control theories are highly sensitive to system's behavior and work based on cancelling decoupling and nonlinear terms of dynamic parameters of each likes in robot manipulators. Computed Torque Control (CTC) and Sliding Mode Control (SMC) are two nonlinear conventional controller which introduced by many researchers to control of OCTARM continuum robot manipulator [17]. 
Computed Torque Controller (CTC) is one of the effective nonlinear controllers to control of robot manipulator. Consequently, to have a good performance, linearization and decoupling without using many gears, feedback linearization (computed-torque) control methodologies is presented. To design computed torque controller, an accurate dynamic model of robot manipulator plays an important role. To modelling an accurate dynamic system, modelling of complex parameters is needed to form the structure of systems dynamic model. It may be very difficult to include all the complexities in the system dynamic model [18]. Dynamic parameters may not be constant over time and measure the

Acceleration term should be measured is very expensive, thus this problem is the main challenge to select the computed torque controller as the main controller in this research. To solve pure computed torque controller, intelligent computed torque controller such as fuzzy computed torque controller [19] or other type of conventional controller such as sliding mode controller is recommended. In this research to design robust control of robot manipulator sliding mode controller is recommended.

To eliminate the actual acceleration measurement and also the computation burden as well as have stability, efficiency and robust controller, sliding mode controller is introduced in this part. This controller works very well in certain and partly uncertain condition [18-20]. This controller has two important subparts, switching part and equivalent part. Switching part of controller is used to design suitable tracking performance based on very fast switching. This part has essential role to have a good trajectory performance in all joints. However this part is very important in uncertain condition but it is caused to chattering phenomenon in system performance. Chattering phenomenon can cause some important mechanical problems [18]. The second subpart in sliding mode controller is equivalent part especially in uncertain condition. Sliding mod controller is a nonlinear model based controller and equivalent part is a dynamic formulation of robot manipulator which is used in control formulation of robot manipulator which is used in control formulation to eliminate the decoupling and nonlinear term of dynamic parameters of each link [19]. However this part is very essential to reliability but in uncertain condition or highly nonlinear dynamic systems it can cause some problem. However conventional sliding mode controller is a robust, stable and reliable controller but there are three main issues limiting; equivalent part related to dynamic equation of robot manipulator, computation of the bounds of uncertainties and chattering phenomenon [20].

The problem in equivalent nonlinear dynamic formulation of robot manipulator is not a simple task and in this research, baseline-adaptive method is used to reduce this challenge. Due to literature to reduce or eliminate the chattering two main methodologies are introduced [17-20]:

- Linear (saturation) boundary layer method

- Nonlinear artificial intelligence based method

However eliminating the switching discountinous function in sliding mode controllers are used in many research but it can causes to lost the robustness of control and accuracy. Uncertainties are very important challenges and caused to overestimation of the bounds. As this point if the sliding surface is equal to zero and it is chosen as desired sliding surface, the dynamic of robot manipulator is derived to sliding surface and if switching function is used to reduce the challenge of uncertainty then the linearization and decoupling through the use of feedback, not gears, can be realized.

This paper is organized as follows; section 2, is served as an introduction to the dynamic of continuum robot and robust nonlinear controller. Part 3 introduces and describes the methodology algorithm. Section 4 presents the simulation results and discussion of this algorithm applied to a continuum robot and the last part is described as conclusion. 


\section{Theory}

Dynamic Formulation of Continuum Robot: The Continuum section analytical model developed here consists of three modules stacked together in series. In general, the model will be a more precise replication of the behavior of a continuum arm with a greater of modules included in series. However, we will show that three modules effectively represent the dynamic behavior of the hardware, so more complex models are not motivated. Thus, the constant curvature bend exhibited by the section is incorporated inherently within the model. The model resulting from the application of Lagrange's equations of motion obtained for this system can be represented in the form

$$
\boldsymbol{F}_{\text {coeff }} \underline{\boldsymbol{\tau}}=\boldsymbol{D}(\underline{\boldsymbol{q}}) \underline{\ddot{q}}+\boldsymbol{C}(\underline{\boldsymbol{q}}) \underline{\dot{q}}+\boldsymbol{G}(\underline{\boldsymbol{q}})
$$

Where $\tau$ a vector of input forces and $\mathrm{q}$ is is a vector of generalized co-ordinates. The force coefficient matrix $F_{\text {coeff }}$ transforms the input forces to the generalized forces and torques in the system. The inertia matrix, $D$ is composed of four block matrices. The block matrices that correspond to pure linear accelerations and pure angular accelerations in the system (on the top left and on the bottom right) are symmetric. The matrix $C$ contains coefficients of the first order derivatives of the generalized co-ordinates. Since the system is nonlinear, many elements of $C$ contain first order derivatives of the generalized co-ordinates. The remaining terms in the dynamic equations resulting from gravitational potential energies and spring energies are collected in the matrix $G$. The coefficient matrices of the dynamic equations are given below,

\section{Fcoeff $=$}

$$
\left[\begin{array}{cc}
1 & 1 \\
0 & 0 \\
0 & 0 \\
1 / 2 & -1 / 2 \\
0 & 0 \\
0 & 0
\end{array}\right.
$$

$\begin{array}{cc}\cos \left(\theta_{1}\right) & \cos \left(\theta_{1}\right) \\ 1 & 1 \\ 0 & 0 \\ 1 / 2 & -1 / 2 \\ 1 / 2 & -1 / 2 \\ 0 & 0\end{array}$

$$
\begin{array}{cr}
\cos \left(\theta_{1}+\theta_{2}\right) & \cos \left(\theta_{1}\right. \\
\cos \left(\theta_{2}\right) & \cos \left(\theta_{2}\right) \\
1 & 1
\end{array}
$$

$$
\begin{gathered}
\cos \left(\theta_{1}+\theta_{2}\right) \\
\cos \left(\theta_{2}\right) \\
1
\end{gathered}
$$

$$
\begin{array}{ll}
1 / 2 & -1 / 2 \\
1 / 2 & -1 / 2
\end{array}
$$

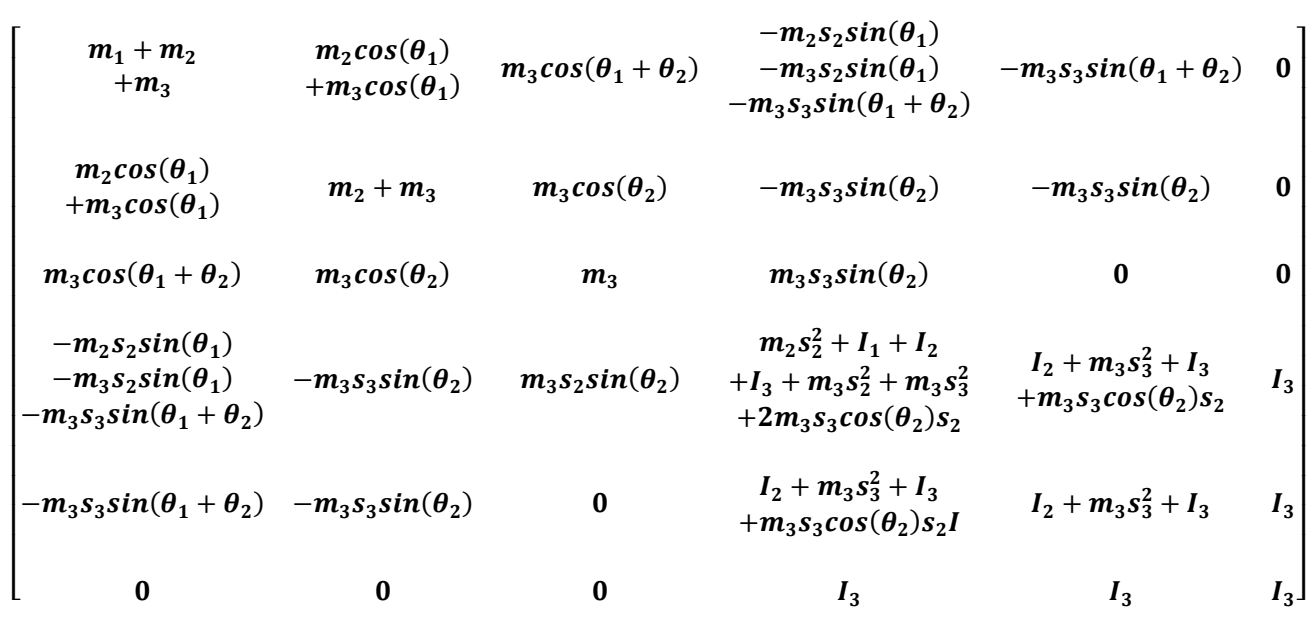




$$
\begin{aligned}
& (\underline{q})=
\end{aligned}
$$

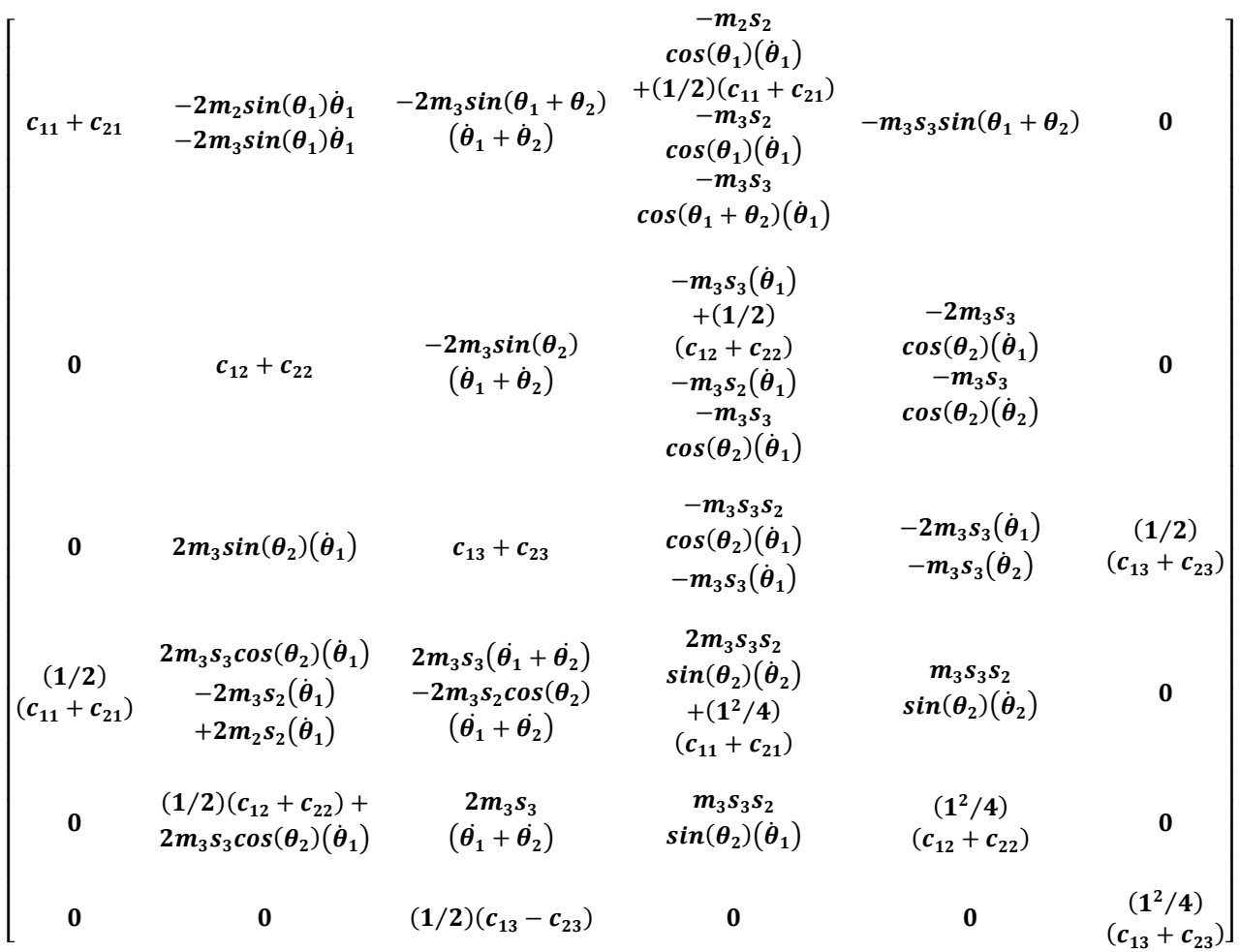

$$
\begin{aligned}
& \boldsymbol{G}(\underline{\boldsymbol{q}})= \\
& {\left[\begin{array}{c}
-m_{1} g-m_{2} g+k_{11}\left(s_{1}+(1 / 2) \theta_{1}-s_{01}\right)+k_{21}\left(s_{1}-(1 / 2) \theta_{1}-s_{01}\right)-m_{3} g \\
-m_{2} g \cos \left(\theta_{1}\right)+k_{12}\left(s_{2}+(1 / 2) \theta_{2}-s_{02}\right)+k_{22}\left(s_{2}-(1 / 2) \theta_{2}-s_{02}\right)-m_{3} g \cos \left(\theta_{1}\right) \\
-m_{3} g \cos \left(\theta_{1}+\theta_{2}\right)+k_{13}\left(s_{3}+(1 / 2) \theta_{3}-s_{03}\right)+k_{23}\left(s_{3}-(1 / 2) \theta_{3}-s_{03}\right) \\
m_{2} s_{2} g \sin \left(\theta_{1}\right)+m_{3} s_{3} g \sin \left(\theta_{1}+\theta_{2}\right)+m_{3} s_{2} g \sin \left(\theta_{1}\right)+k_{11}\left(s_{1}+(1 / 2) \theta_{1}-s_{01}\right)(1 / 2) \\
+k_{21}\left(s_{1}-(1 / 2) \theta_{1}-s_{01}\right)(-1 / 2) \\
m_{3} s_{3} g \sin \left(\theta_{1}+\theta_{2}\right)+k_{12}\left(s_{2}+(1 / 2) \theta_{2}-s_{02}\right)(1 / 2)+k_{22}\left(s_{2}-(1 / 2) \theta_{2}-s_{02}\right)(-1 / 2) \\
k_{13}\left(s_{3}+(1 / 2) \theta_{3}-s_{03}\right)(1 / 2)+k_{23}\left(s_{3}-(1 / 2) \theta_{3}-s_{03}\right)(-1 / 2)
\end{array}\right]}
\end{aligned}
$$

Sliding Mode Controller: Consider a nonlinear single input dynamic system is defined by [6]:

$$
x^{(n)}=f(\vec{x})+b(\vec{x}) u
$$

Where $\mathrm{u}$ is the vector of control input, $\boldsymbol{x}^{(\boldsymbol{n})}$ is the $\boldsymbol{n}^{\text {th }}$ derivation of $\boldsymbol{x}, \boldsymbol{x}=$ $\left[\boldsymbol{x}, \dot{\boldsymbol{x}}, \ddot{\boldsymbol{x}}, \ldots, \boldsymbol{x}^{(\boldsymbol{n}-1)}\right]^{T}$ is the state vector, $\boldsymbol{f}(\boldsymbol{x})$ is unknown or uncertainty, and $\boldsymbol{b}(\boldsymbol{x})$ is of known sign function. The main goal to design this controller is train to the desired state; $x_{d}=\left[x_{d}, \dot{x}_{d}, \ddot{x}_{d}, \ldots, x_{d}^{(n-1)}\right]^{T}$, and trucking error vector is defined by [6]:

$$
\widetilde{x}=x-x_{d}=\left[\widetilde{x}, \ldots, \widetilde{x}^{(n-1)}\right]^{T}
$$

A time-varying sliding surface $\boldsymbol{s}(\boldsymbol{x}, \boldsymbol{t})$ in the state space $\boldsymbol{R}^{\boldsymbol{n}}$ is given by [20]:

$$
s(x, t)=\left(\frac{d}{d t}+\lambda\right)^{n-1} \widetilde{x}=0
$$


Where $\lambda$ is the positive constant. To further penalize tracking error, integral part can be used in sliding surface part as follows [20]:

$$
\boldsymbol{s}(\boldsymbol{x}, \boldsymbol{t})=\left(\frac{d}{d t}+\lambda\right)^{n-1}\left(\int_{0}^{t} \widetilde{\boldsymbol{x}} \boldsymbol{d t}\right)=\mathbf{0}
$$

The main target in this methodology is kept the sliding surface slope $\boldsymbol{s}(\boldsymbol{x}, \boldsymbol{t})$ near to the zero. Therefore, one of the common strategies is to find input $\boldsymbol{U}$ outside of $\boldsymbol{s}(\boldsymbol{x}, \boldsymbol{t})$ [11].

$$
\frac{1}{2} \frac{d}{d t} s^{2}(x, t) \leq-\zeta|s(x, t)|
$$

Where $\zeta$ is positive constant.

$$
\text { If } \mathbf{S}(\mathbf{0})>\mathbf{0} \rightarrow \frac{\mathrm{d}}{\mathrm{dt}} \mathbf{S}(\mathbf{t}) \leq-\zeta
$$

To eliminate the derivative term, it is used an integral term from $\mathrm{t}=0$ to $\mathrm{t}=\boldsymbol{t}_{\text {reach }}$

$$
\int_{t=0}^{t=t_{\text {reach }}} \frac{d}{d t} S(t) \leq-\int_{t=0}^{t=t_{\text {reach }}} \eta \rightarrow S\left(t_{\text {reach }}\right)-S(0) \leq-\zeta\left(t_{\text {reach }}-0\right)
$$

Where $t_{\text {reach }}$ is the time that trajectories reach to the sliding surface so, suppose $\mathrm{S}$ $\left(t_{\text {reach }}=0\right)$ defined as;

$$
0-S(0) \leq-\eta\left(t_{\text {reach }}\right) \rightarrow t_{\text {reach }} \leq \frac{S(0)}{\zeta}
$$

And

$$
\text { if } \boldsymbol{S}(\mathbf{0})<0 \rightarrow 0-S(\mathbf{0}) \leq-\eta\left(\boldsymbol{t}_{\text {reach }}\right) \rightarrow \boldsymbol{S}(\mathbf{0}) \leq-\zeta\left(\boldsymbol{t}_{\text {reach }}\right) \rightarrow \boldsymbol{t}_{\text {reach }} \leq \frac{|\boldsymbol{S}(\mathbf{0})|}{\eta}
$$

Equation (14) guarantees time to reach the sliding surface is smaller than $\frac{|S(\mathbf{0})|}{\zeta}$ since the trajectories are outside of $S(t)$.

$$
\text { if } S_{t_{\text {reach }}}=S(0) \rightarrow \operatorname{error}\left(x-x_{d}\right)=0
$$

Suppose $\mathrm{S}$ is defined as

$$
s(x, t)=\left(\frac{d}{d t}+\lambda\right) \quad \tilde{x}=\left(\dot{\mathbf{x}}-\dot{\mathbf{x}}_{\mathbf{d}}\right)+\lambda\left(\mathbf{x}-\mathbf{x}_{\mathbf{d}}\right)
$$

The derivation of S, namely, $\dot{S}$ can be calculated as the following;

$$
\dot{\boldsymbol{S}}=\left(\ddot{\mathbf{x}}-\ddot{\mathbf{x}}_{\mathbf{d}}\right)+\boldsymbol{\lambda}\left(\dot{\mathbf{x}}-\dot{\mathbf{x}}_{\mathbf{d}}\right)
$$

Suppose the second order system is defined as;

$$
\ddot{\boldsymbol{x}}=\boldsymbol{f}+\boldsymbol{u} \rightarrow \dot{\boldsymbol{S}}=\boldsymbol{f}+\boldsymbol{U}-\ddot{\boldsymbol{x}}_{\boldsymbol{d}}+\boldsymbol{\lambda}\left(\dot{\mathrm{x}}-\dot{\mathbf{x}}_{\mathrm{d}}\right)
$$

Where $\boldsymbol{f}$ is the dynamic uncertain, and also since $S=0$ and $\dot{S}=0$, to have the best approximation, $\widehat{\boldsymbol{U}}$ is defined as

$$
\widehat{\boldsymbol{U}}=-\widehat{\boldsymbol{f}}+\ddot{\boldsymbol{x}}_{\boldsymbol{d}}-\lambda\left(\dot{\mathbf{x}}-\dot{\mathbf{x}}_{\mathbf{d}}\right)
$$

A simple solution to get the sliding condition when the dynamic parameters have uncertainty is the switching control law [11-13]:

$$
U_{d i s}=\widehat{U}-K(\vec{x}, t) \cdot \operatorname{sgn}(s)
$$

Where the switching function $\mathbf{s g n}(\mathbf{S})$ is defined as [20] 


$$
\operatorname{sgn}(s)= \begin{cases}1 & s>0 \\ -1 & s<0 \\ 0 & s=0\end{cases}
$$

And the $\boldsymbol{K}(\overrightarrow{\boldsymbol{x}}, \boldsymbol{t})$ is the positive constant. Suppose by (10) the following equation can be written as,

$$
\frac{1}{2} \frac{d}{d t} s^{2}(x, t)=\dot{S} \cdot S=[f-\widehat{f}-K \operatorname{sgn}(s)] \cdot S=(f-\widehat{f}) \cdot S-K|S|
$$

And if the equation (14) instead of (13) the sliding surface can be calculated as

$$
s(x, t)=\left(\frac{d}{d t}+\lambda\right)^{2}\left(\int_{0}^{t} \tilde{x} d t\right)=\left(\dot{x}-\dot{x}_{d}\right)+2 \lambda\left(\dot{x}-\dot{x}_{d}\right)-\lambda^{2}\left(x-x_{d}\right)
$$

In this method the approximation of $\boldsymbol{U}$ is computed as [6]

$$
\widehat{U}=-\widehat{f}+\ddot{x}_{d}-2 \lambda\left(\dot{\mathbf{x}}-\dot{\mathbf{x}}_{\mathbf{d}}\right)+\lambda^{2}\left(\mathbf{x}-\mathbf{x}_{\mathbf{d}}\right)
$$

Based on above discussion, the variable structure control law for a multi degrees of freedom robot manipulator is written as [7]:

$$
\tau=\tau_{e q}+\tau_{d i s}
$$

Where, the model-based component $\boldsymbol{\tau}_{\boldsymbol{e q}}$ is the nominal dynamics of systems calculated as follows [1-3]:

$$
\tau_{e q}=\left[M^{-1}(B+C+G)+\dot{S}\right] M
$$

And $\boldsymbol{\tau}_{\text {dis }}$ is computed as [11-15];

$$
\tau_{d i s}=K \cdot \operatorname{sgn}(S)
$$

By (27) and (26) the variable structure control of robot manipulator is calculated as;

$$
\tau=\left[M^{-1}(B+C+G)+\dot{S}\right] M+K \cdot \operatorname{sgn}(S)
$$

The lyapunov formulation can be written as follows,

$$
V=\frac{1}{2} S^{T} . M . S
$$

The derivation of $V$ can be determined as,

$$
\dot{V}=\frac{1}{2} S^{T} \cdot \dot{M} \cdot S+S^{T} M \dot{S}
$$

The dynamic equation of robot manipulator can be written based on the sliding surface as

$$
M \dot{S}=-V S+M \dot{S}+B+C+G
$$

It is assumed that

$$
S^{T}(\dot{M}-2 B+C+G) S=0
$$

By substituting (31) in (32)

$$
\dot{V}=\frac{1}{2} S^{T} \dot{M} S-S^{T} B+C S+S^{T}(M \dot{S}+B+C S+G)=S^{T}(M \dot{S}+B+C S+G)
$$

Suppose the control input is written as follows

$$
\widehat{U}=U_{\text {Nonlınear }}+\widehat{U_{d l s}}=\left[\widehat{M^{-1}}(B+C+G)+\dot{S}\right] \widehat{M}+K \cdot \operatorname{sgn}(S)+B+C S+G
$$

By replacing the equation (37) in (29) 


$$
\begin{aligned}
\dot{V}= & S^{T}(M \dot{S}+B+C+G-\widehat{M} \dot{S}-\widehat{B+C S}+G-K \operatorname{sgn}(S) \\
& =S^{T}(\widetilde{M} \dot{S}+\widehat{B+C S}+G-K \operatorname{sgn}(S))
\end{aligned}
$$

And

$$
|\widetilde{M} \dot{S}+\widetilde{B+C S}+G| \leq|\widetilde{M} \dot{S}|+|\widetilde{B+C S}+G|
$$

The Lemma equation in robot arm system can be written as follows

$$
K_{u}=[|\widetilde{M} \dot{S}|+|B+C S+G|+\eta]_{i}, i=1,2,3,4, \ldots
$$

and finally;

$$
\dot{V} \leq-\sum_{i=1}^{n} \eta_{i}\left|S_{i}\right|
$$

\section{Methodology}

In sliding mode controller select the desired sliding surface and sign function play a vital role to system performance and if the dynamic of robot manipulator is derived to sliding surface then the linearization and decoupling through the use of feedback, not gears, can be realized. In this state, the derivative of sliding surface can help to decoupled and linearized closed-loop OCTARM continuum robot dynamics that one expects in computed torque control. Linearization and decoupling by sliding mode controller can be obtained in spite of the quality of the robot manipulator dynamic model, in contrast to the computed-torque control that requires the exact dynamic model of a system. As a result, uncertainties are estimated by discontinuous feedback control but it can cause to chattering. To reduce the chattering in presence of switching functions; linear controller is added to discontinuous part of sliding mode controller. Linear controller is type of stable controller as well as conventional sliding mode controller. In proposed methodology PD, PI or PID linear controller is used in parallel with discontinuous part to reduce the role of sliding surface slope as a main coefficient. The formulation of new chattering free sliding mode controller for robot manipulator is;

$$
\tau=\tau_{e q}+\tau_{\text {dis-new }}
$$

In (39) $\boldsymbol{\tau}_{\boldsymbol{e q}}$ is equivalent term of sliding mode controller and this term is related to the nonlinear dynamic formulation of robot manipulator. The new switching discontinuous part is introduced by $\boldsymbol{\tau}_{\text {dis-new }}$ a6nd this item is the important factor to resistance and robust in this controller. In PD sliding surface, based on the change of sliding surface calculated as;

$$
S_{P D}=\lambda e+\dot{e} \rightarrow \dot{S}_{P D}=\lambda \dot{e}+\ddot{e}
$$

The discontinuous switching term ( $\left.\boldsymbol{\tau}_{\text {dis }}\right)$ is computed as;

$$
\tau_{\text {dis-new }}=K_{a} \cdot \operatorname{sgn}(S)+K_{b} \cdot S
$$

Based on (41) and (40);

$$
\tau_{\text {dis-PD-new }}=K_{a} \cdot \operatorname{sgn}(\lambda e+\dot{e})+K_{b} \cdot(\lambda e+\dot{e})
$$

According to (41) and (39);

$$
\tau_{d i s-P I-n e w}=K_{a} \cdot \operatorname{sgn}\left(\lambda e+\left(\frac{\lambda}{2}\right)^{2} \sum e\right)+K_{b} \cdot\left(\lambda e+\left(\frac{\lambda}{2}\right)^{2} \sum e\right)
$$

By replace (40) in (41) the discontinuous switching part is; 


$$
\tau_{d i s-P I D-n e w}=K_{a} \cdot \operatorname{sgn}\left(\lambda e+\dot{e}+\left(\frac{\lambda}{2}\right)^{2} \sum e\right)+K_{b} \cdot\left(\lambda e+\dot{e}+\left(\frac{\lambda}{2}\right)^{2} \sum e\right)
$$

According to (44) and (41);

$$
\begin{gathered}
\tau=\tau_{e q}+K_{a} \cdot \operatorname{sgn}(S)+K_{b} \cdot S \\
=\left[A^{-1}(q) \times(N(q, \dot{q}))+\dot{S}\right] \times A(q)+K_{a} \cdot \operatorname{sgn}(S)+K_{b} \cdot S
\end{gathered}
$$

According to (42) and (45) the formulation of PD-SMC is;

$$
\begin{aligned}
\tau_{P D-S M C-n e w} & =K_{a} \cdot \operatorname{sgn}(\lambda e+\dot{e})+K_{b} \cdot(\lambda e+\dot{e})+\left[A^{-1}(q) \times(N(q, \dot{q}))+\dot{S}\right] \\
& \times A(q)
\end{aligned}
$$

According to the dynamic formulation of robot manipulator

$$
\tau=A(q) \ddot{q}+V(q, \dot{q}) \dot{q}+G(q)
$$

And the controller formulation

$$
\boldsymbol{\tau}=\widehat{A} \ddot{\boldsymbol{q}}_{r}+\widehat{V} \dot{\boldsymbol{q}}_{r}+\widehat{\boldsymbol{G}}+\boldsymbol{K} \boldsymbol{s g n}(\boldsymbol{S})+\boldsymbol{K} \cdot \boldsymbol{S}
$$

According to (47) and (48), can be expressed:

$$
\boldsymbol{A}(\boldsymbol{q}) \ddot{\boldsymbol{q}}+\boldsymbol{V}(\boldsymbol{q}, \dot{\boldsymbol{q}}) \dot{\boldsymbol{q}}+\boldsymbol{G}(\boldsymbol{q})=\widehat{A} \ddot{\boldsymbol{q}}_{r}+\widehat{V} \dot{\boldsymbol{q}}_{r}+\widehat{\boldsymbol{G}}+K_{a} \operatorname{sgn}(\boldsymbol{S})+K_{b} . S
$$

Where $\widehat{A}, \widehat{V}$ and $\widehat{G}$ are the estimation of $\boldsymbol{A}(\boldsymbol{q}), \boldsymbol{V}(\boldsymbol{q}, \dot{\boldsymbol{q}})$ and $\boldsymbol{G}(\boldsymbol{q})$ and in this formulation?

$K_{a}=\left[K_{a_{1}}, K_{a_{2}}, K_{a_{3}}, K_{a_{4}}, K_{a_{5}}, K_{a_{6}}\right]$ and $K_{b}=\left[K_{b_{1}}, K_{b_{2}}, K_{b_{3}}, K_{b_{4}}, K_{b_{5}}, K_{b_{6}}\right]$

Since $\dot{\boldsymbol{q}}_{\boldsymbol{d}}=\dot{\boldsymbol{q}}-\boldsymbol{S}$ and $\ddot{\boldsymbol{q}}_{\boldsymbol{d}}=\ddot{\boldsymbol{q}}-\dot{\boldsymbol{S}}$

$$
A \dot{S}+\left(V+K_{b}\right) S=\Delta f-K_{a} \operatorname{sgn}(S)
$$

Where $\Delta \boldsymbol{f}=\Delta \boldsymbol{A} \ddot{\boldsymbol{q}}+\Delta \boldsymbol{V} \dot{\boldsymbol{q}}+\Delta \boldsymbol{G}$ and $\Delta \boldsymbol{A}=\widehat{\boldsymbol{A}}-\boldsymbol{A}, \Delta \boldsymbol{V}=\widehat{\boldsymbol{V}}-\boldsymbol{V}$ and $\Delta \boldsymbol{G}=\widehat{\boldsymbol{G}}-\boldsymbol{G}$

The dynamic equation of robot manipulator can be written based on the sliding surface as

$$
A \dot{S}=-V S+A \dot{S}+V S+G-\tau
$$

Assuming that it can be expressed by the following equation:

$$
S^{T}(\dot{A}-2 V) S=0
$$

If the Lyaponuv function is written by;

$$
V=\frac{1}{2} S^{T} A(q) S
$$

According to (52) we can be written the derivative of Lyapunov functions as;

$$
\begin{gathered}
\dot{V}=S^{T} A \dot{S}+\frac{1}{2} S^{T} \dot{A} S \\
=S^{T}(A \dot{S}+V S) \\
=S^{T}\left[-k_{a b} S+\Delta f-k_{a} \operatorname{sgn}(S)\right] \\
=\sum_{i=1}^{6}\left(S_{i}\left[\Delta f_{i}-k_{a i} \operatorname{sgn}\left(S_{i}\right)\right]\right)-S^{T} k_{b} S .
\end{gathered}
$$

And with regard to substituting (52) in (54); 


$$
\dot{V}=\frac{1}{2} S^{T} \dot{A} S-S^{T} V S+S^{T}(A \dot{S}+V S+G-\tau)=S^{T}(A \dot{S}+V S+G-\tau)
$$

According to the sliding mode controller formulation;

$$
\widehat{\tau}=\widehat{\tau_{e q}}+\widehat{\tau_{d l s}}=\left[\widehat{A^{-1}}(\widehat{V}+\widehat{G})+\dot{S}\right] \widehat{A}+K_{a} \operatorname{sgn}(S)+K_{b} \cdot S
$$

By replacing the equation (56) in (55)

$$
\begin{array}{r}
\dot{V}=S^{T}\left(A \dot{S}+V S+G-\widehat{A} \dot{S}-\widehat{V} S-\widehat{G}-K_{a} \operatorname{sgn}(S)-K_{b} . S\right. \\
=S^{T}\left(\widetilde{A} \dot{S}+\widetilde{V} S+\widetilde{G}-K \operatorname{sgn}(S)-K_{b} . S\right)
\end{array}
$$

For $k_{a i} \geq\left|\Delta f_{i}\right|$ we always get $S_{i}\left[\Delta \boldsymbol{f}_{\boldsymbol{i}}-\boldsymbol{k}_{\boldsymbol{a} i} \boldsymbol{s} \boldsymbol{g n}\left(\boldsymbol{S}_{\boldsymbol{i}}\right)\right] \leq \mathbf{0}$ and we can write:

$$
\dot{V}=\sum_{i=1}^{6}\left(S_{i}\left[\Delta f_{i}-k_{a i} \operatorname{sgn}\left(S_{i}\right)\right]\right)-S^{T} k_{b i} S \leq-S^{T} k_{b i} S<0 \quad(S \neq 0)
$$

The modify vibration free sliding mode controller can be updated based on online tuning sliding surface slope. In order to reduce the online computation burden, the baseline controller is also used to sliding surface slope online tuning.

Baseline controller is used to increase the system stability in presence of system uncertainty.

$$
\begin{gathered}
S_{P D}=\lambda e+\dot{e} \\
\lambda_{\text {update }}=\lambda \times U_{\text {Baseline }} \\
\lambda_{\text {update }}=\left(\lambda U_{P I D}+\frac{A S}{S+B} \times U_{P I D}\right)
\end{gathered}
$$

Where $\lambda_{\text {update }}$ is online tuning of sliding surface slope using baseline controller. According to (60) the modify online tuning sliding surface slope sliding mode controller is;

$$
\begin{aligned}
& \tau_{\text {Modifyvibration free SMC-new }} \\
& \qquad \begin{aligned}
K_{a} \cdot \operatorname{sgn}\left(\lambda_{\text {update }} e+\dot{e}\right)+K_{b} \cdot\left(\lambda_{\text {update }} e+\dot{e}\right) \\
+\left(\left[\widehat{A}^{-1}(q) \times(\widehat{N}(q, \dot{q}))+\dot{S}\right] \times \widehat{A}(q)\right.
\end{aligned}
\end{aligned}
$$

\section{Results and Discussion}

Vibration reduction and rise-time: The trajectory following for online sliding surface slope vibration free sliding mode controller, vibration free sliding mode controller and boundary layer sliding mode controller are compared in Figure 1. Based on the Figure 1, all three controllers can eliminate the chattering and vibration in certain situation. In rise time point of view, online sliding surface slope vibration free sliding mode controller is faster (0.48 second) than vibration frees sliding mode controller (0.6 second) and boundary layer sliding mode controller ( 2.1 second). 


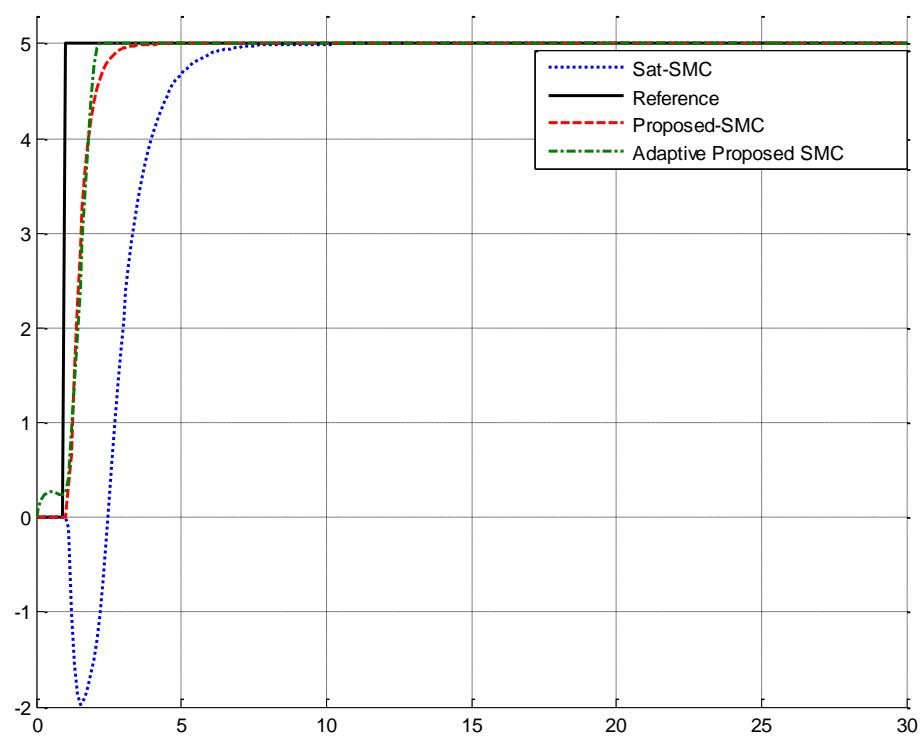

Figure 1. Saturation SMC, Parallel Linear SMC (Proposed-SMC) and Adaptive Proposed-SMC

Eliminate the vibration and robustness: the power of disturbance rejection is very important to robust checking in any controllers. In this part, trajectory accuracy is test under uncertainty condition. To test the disturbance rejection band limited white noise with $30 \%$ amplitude is applied to online sliding surface slope vibration free sliding mode controller, vibration free sliding mode controller and boundary layer sliding mode controller. According to Figure 2, however parallel linear sliding mode controller has suitable steady state error in presence of uncertainty but it is more robust than boundary layer sliding mode controller. Boundary layer sliding mode controller has very much fluctuations in presence of external disturbance. Regarding to the following Figure, online sliding surface slope vibration free sliding mode controller have the power of eliminate the vibration in certain and uncertain condition. This control technique has acceptable rise time in certain and uncertain condition.

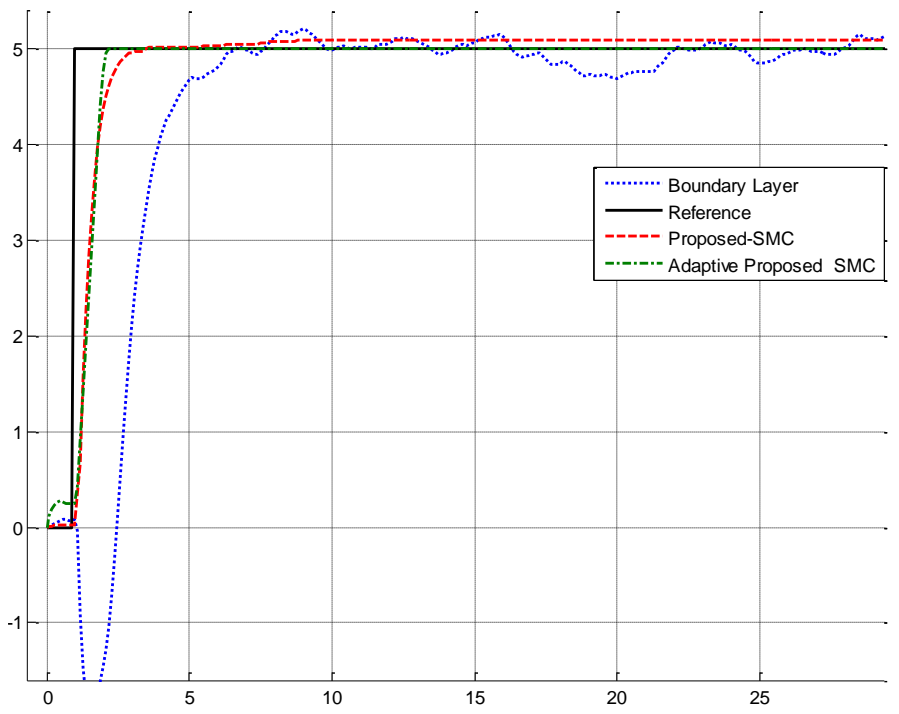

Figure 2: Comparison of Disturbance Rejection: Parallel Linear SMC (Proposed-SMC), Boundary Layer Method and Adaptive Proposed-SMC in Presence of Uncertainty 


\section{Conclusion}

In this research, on-line sliding surface slope tuning vibration free sliding mode controller is recommended for continuum robot manipulator. To design robust and stable controller conventional sliding mode controller is recommended. Conventional sliding mode controller (SMC) is a nonlinear, stable, robust and reliable controller. This type of controller has two main important challenges: chattering phenomenon and equivalent part related to dynamic equation of robot manipulator. The first challenge to design robust and stable sliding mode controller based on switching function is chattering phenomenon. In sliding mode controller select the desired sliding surface and sign function play a vital role to system performance and if the dynamic of robot manipulator is derived to sliding surface then the linearization and decoupling through the use of feedback, not gears, can be realized. As a result, uncertainties are estimated by discontinuous feedback control but it can cause to chattering. To reduce the chattering in presence of switching functions; linear controller is added to discontinuous part of sliding mode controller. Linear controller is type of stable controller as well as conventional sliding mode controller. In proposed methodology PD, PI or PID linear controller is used in parallel with discontinuous part to reduce the role of sliding surface slope as a main coefficient. Conventional sliding mode controller is worked based on manipulator dynamic model. Based on equivalent part in conventional nonlinear controllers, in complex and highly nonlinear systems these controllers have many problems for accurate responses because these type of controllers need to have accurate knowledge of dynamic formulation of system. The nonlinear dynamic formulation problem in highly nonlinear system (e.g., robot manipulator) can be solved by online tuning baseline method. This type of controller is free of mathematical dynamic parameters of plant. When system works in uncertainty, the nonlinearity term of robot manipulator is not equal to equivalent term of sliding mode controller. To reduce the role of nonlinearity term of SMC, baseline controller is used in this research. The simulation results show that the proposed controller works well in various situations. This type of method is the best robust method to eliminate the vibration in presence of uncertainty with switching function. Based on result and discussion, proposed method can eliminate vibration in certain and uncertain condition.

\section{Acknowledgments}

The authors would like to thank the anonymous reviewers for their careful reading of this paper and for their helpful comments. This work was supported by the Iranian Institute of Advance Science and Technology Program of Iran under grant no. 2012Persian Gulf-2A.

Iranian center of Advance Science and Technology (IRAN SSP) is one of the independent research centers specializing in research and training across of Control and Automation, Electrical and Electronic Engineering, and Mechatronics \& Robotics in Iran. At IRAN SSP research center, we are united and energized by one mission to discover and develop innovative engineering methodology that solve the most important challenges in field of advance science and technology. The IRAN SSP Center is instead to fill a long standing void in applied engineering by linking the training a development function one side and policy research on the other. This center divided into two main units:

- $\quad$ Education unit

- $\quad$ Research and Development unit 


\section{References}

[1] Vachtsevanos, G. I., Davey, K. and Lee, K. M., "Development of a Novel Intelligent Robotic Manipulator," IEEE Control System Magazine, 1987, pp.9-15.

[2] Davey, K., Vachtsevanos, G. I., and Powers, R., "An analysis of Fields and Torques in Spherical Induction Motors," IEEE Transactions on Magnetics, Vol. MAG-23, 1987, pp. 273-282.

[3] Foggia, A., Oliver, E., Chappuis, F., "New Three Degrees of Freedom Electromagnetic Actuator," Conference Record -lAS Annual Meeting, Vol. 35, New York, 1988.

[4] Lee, K. M., Vachtsevanos, G. and Kwan, C-K., "Development of a Spherical Wrist Stepper Motor," Proceedings of the 1988 IEEE lntemational Conference on Robotics and Automation, Philadelphia, PA. April 26-29.

[5] Lee, K. M., Pei. I., "Kinematic Analysis of a Three Degree-of-Freedom Spherical Wrist Actuator," The Fifth International Conference on Advanced Robotics, Italy,1991.

[6] Wang, I., Jewel, G., Howe, D., "Modeling of a Novel Spherical Pennanent Magnet Actuator," Proceedings of IEEE International Conference on Robotics and Automation, Albuquerque, New Mexico, pp 1190-1195, 1997.

[7] Wang, I., Jewel, G., Howe, D., "Analysis, Design and Control of a Novel Spherical Pennanent Magnet Actuator," 1EE Proceedings on Electrical Power Applications., vol. 154, no. 1, 1998.

[8] Chirikjian, G. S., and Stein, D., "Kinematic Design and Commutation of a Spherical Stepper Motor," IEEEIASME Transactions on Mechatronics, vol. 4, n 4, Piscataway, New Jersey, pp. 342-353, Dec. 1999.

[9] Kahlen, K., and De Doncker, R. W., "CW'l'ent Regulators for Multi-phase Pennanent Magnet Spherical Machines." Industry Applications Conference Record of the 2000 IEEE, vol. 3, 2000, pp. 2011-2016.

[10] Lee, K. M., Pei, I., and Gilboa, U., "On the Development of a Spherical Wrist Actuator," Proceedings of the 16th NSF Conference on Manufacturing Systems Research, Tempe AZ, January 8-12, 1990.

[11] Z. Liu, H. Su, S. Pan, A new adaptive sliding mode control of uncertain nonlinear systems, Asian Journal of Control, 2014, vol. 16, no. 1, 198-208

[12] Y. Shang, Consensus recovery from intentional attacks in directed nonlinear multi-agent systems, International Journal of Nonlinear Sciences and Numerical Simulation, 2013, vol. 14, no. 6, 355-361

[13] Farzin Piltan, A. R. Salehi \& Nasri B Sulaiman,"Design Artificial Robust Control of Second Order System Based on Adaptive Fuzzy Gain Scheduling", World Applied Science Journal (WASJ), 13 (5): 1085-1092, 2011, (ISI, Scopus, SJR=0.22, Q2)M. N. Kamarudin, A. R. Husain, M. N. Ahmad, Control of uncertain nonlinear systems using mixed nonlinear damping function and backstepping techniques, 2012 IEEE International Conference on Control Systems, Computing and Engineering, Malaysia, 2012

[14] Farzin Piltan, N. Sulaiman, S.Soltani, M. H. Marhaban \& R. Ramli, "An Adaptive Sliding Surface Slope Adjustment in PD Sliding Mode Fuzzy Control For Robot Manipulator", International Journal of Control and Automation, 4 (3): 65-76, 2011, (Scopus, SJR=0.25., Q3)

[15] Alireza Siahbazi, Ali Barzegar, Mahmood Vosoogh, Abdol Majid Mirshekaran, Samira Soltani,"Design Modified Sliding Mode Controller with Parallel Fuzzy Inference System Compensator to Control of Spherical Motor", IJISA, vol.6, no.3, pp.12-25, 2014. DOI: 10.5815/ijisa.2014.03.02

[16] Mojtaba Yaghoot, Farzin Piltan, Meysam Esmaeili, Mohammad Ali Tayebi, Mahsa Piltan,"Design Intelligent Robust Model-base Sliding Guidance Controller for Spherical Motor", IJMECS, vol.6, no.3, pp.61-72, 2014.DOI: 10.5815/ijmecs.2014.03.08

[17] Saman Rahbar, Farzin Piltan, Ehsan Pouladi, Hossein Davarpanah and Somayeh Jowkar," The Fuzzy $(\mathrm{PI}+\mathrm{D})^{2}$ Sliding Mode Scheme to Motor Vibration Control", International Journal of U- and e- Service, Science and Technology, 8(1): 371-388, 2015. http://dx.doi.org/10.14257/ijunesst.2015.8.1.33

[18] Farzin Matin, Farzin Piltan, Hamid Cheraghi, Nasim Sobhani, Maryam Rahmani,"Design Intelligent PID like Fuzzy Sliding Mode Controller for Spherical Motor", IJIEEB, vol.6, no.2, pp.53-63, 2014. DOI: 10.5815/ijieeb.2014.02.07

[19] S.Yauldegar, Hootan Ghiasi, Mohammad Hadi Mazloom, Amirzubir Sahamijoo, Mohammad Reza Avazpour and Farzin Piltan, "Trajectory Tracking Control of Multi Degrees of Freedom Joints: Robust Fuzzy Logic-Based Sliding Mode Approach", International Journal of Control and Automation, 7 (12): 323-338, 2014. (Scopus, SJR=0.25, Q3) 


\section{Authors}
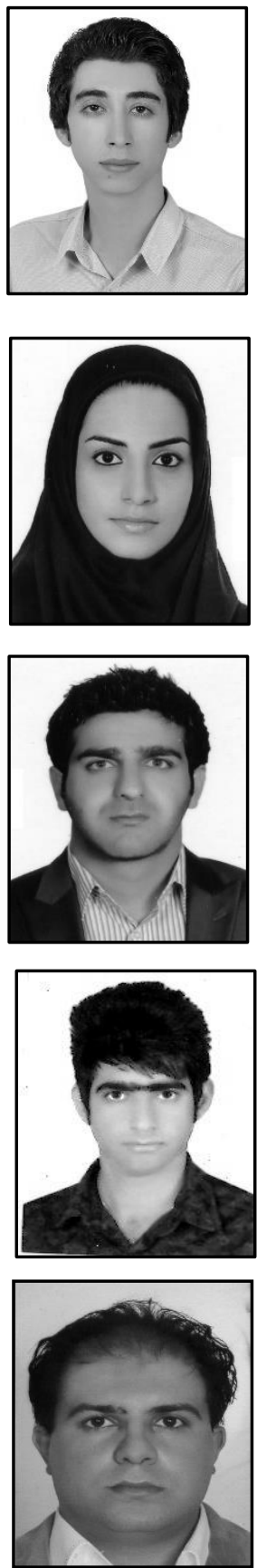

Farzin Piltan was born on 1975, Shiraz, Iran. In 2004 he is jointed Institute of Advance Science and Technology, Research and Development Center, IRAN SSP. Now he is a dean of Intelligent Control and Robotics Lab. He is led of team (47 researchers) to design and build of nonlinear control of industrial robot manipulator for experimental research and education and published about 54 Papers in this field since 2010 to 2012, team supervisor and leader (9 researchers) to design and implement intelligent tuning the rate of fuel ratio in internal combustion engine for experimental research and education and published about 17 Journal papers since 2011 to 2013, team leader and advisor (34 researchers) of filtering the hand tremors in flexible surgical robot for experimental research and education and published about 31 journal papers in this field since 2012 to date, led of team (21 researchers) to design high precision and fast dynamic controller for multi-degrees of freedom actuator for experimental research and education and published about 7 journal papers in this field since 2013 to date, led of team (22 researchers) to research of full digital control for nonlinear systems (e.g., Industrial Robot Manipulator, IC Engine, Continuum Robot, and Spherical Motor) for 
experimental research and education and published about 4 journal papers in this field since 2010 to date and finally led of team (more than 130 researchers) to implementation of Project Based-Learning project at IRAN SSP research center for experimental research and education, and published more than 110 journal papers since 2010 to date. In addition to 7 textbooks, Farzin Piltan is the main author of more than 115 scientific papers in refereed journals. He is editorial review board member for 'international journal of control and automation (IJCA), Australia, ISSN: 2005-4297; 'International Journal of Intelligent System and Applications (IJISA)', Hong Kong, ISSN: 2074-9058; 'IAES international journal of robotics and automation, Malaysia, ISSN: 2089-4856; 'International Journal of Reconfigurable and Embedded Systems', Malaysia, ISSN: 20894864. His current research interests are nonlinear control, artificial control system and applied to FPGA, robotics and artificial nonlinear control and IC engine modeling and control. 
International Journal of Hybrid Information Technology Vol.8, No.7 (2015) 